\title{
La Psicología en España. Ciencia y profesión
}

\section{Helio Carpintero}

En este ciclo pensado en torno al problema del presente y del futuro de la Psicología, me parece que es al menos conveniente, pensar un poco sobre la Psicología en España, porque es justamente el marco, el contexto básico en el que nos tenemos me mover.

Como la evolución de la Psicología en España ha tenido problemas y particularidades que no son sin mas generalizables a otras países, es conveniente que al menos sepamos cuál ha sido la trayectoria de la evolución de nuestra ciencia y también cuáles han sido las resistencias y dificultades con que se ha enfrentado la Psicología en nuestro país, como conocimiento y como profesión, porque las dos dimensiones, las dos y no sólo una, tienen que ser objeto de reflexión por parte de los psicólogos y de la sociedad que se ocupa y se interesa por la Psicología.

Porque, efectivamente, la Psicología es conocimiento, pero es también hoy una profesión, y como tal tiene dimensiones que no se reducen a las de una pura ciencia, sino que implican aspectos de intervención y de aplicabilidad social, y por supuesto dimensiones también de responsabilidad colectiva, que le obligan a hacer frente a problemas que la sociedad tiene hoy y para los cuales la Psicología tiene alguna palabra que decir.

La interacción entre el conocimiento y la profesión no ha sido y todavía no es una relación que esté resuelta suficientemente. Aquí, como ejemplo, me referiré a dos hechos de la Psicología americana. A principios de siglo aquí, James McKeen Cattell quiso y promovió una aplicación de la Psicología a partir de los tests, al mismo tiempo que otro de los grandes psicólogos que entonces estaba en Estados Unidos, E. B. Titchener, decía y se manifestaba en contra de la aplicación de la Psicología, porque le parecía que esa aplicación perturbaba y desnaturalizaba la investigación de laboratorio. Esa oscilación entre una Psicología de laboratorio y una Psicología de compromiso y de aplicación social sigue siendo hoy un problema gravísimo que siguen viviendo los psicólogos de Estados Unidos. Por eso, en la American Psychological Association (APA), hay hoy una visible tensión entre los Psicólogos profesionales y los que llamaríamos académicos, viviendo en una situación en que no está claro si es mejor mantener su unidad, o cambiar y convertirse en dos sociedades, una científica y otra profesional.

Este problema no es, pues, un problema español: es un problema general, el problema que se establece entre el científico investigador y el técnico profesional que aplica el conocimiento a los casos concretos; pero en Psicología ambas dimensiones son fundamentales, y por eso pretendo que ambas dimensiones estén aquí presentes.

La evolución de la Psicología en nuestro país ha sido muy particularmente una evolución doble. Una es la evolución de lo que llamaríamos Psicología aplicada, y otro el desarrollo de la Psicología científica e investigadora. 
Nuestro país ha sido, como otro muchos, un país que ha recibido la Psicología científica. No la hemos inventado nosotros, la hemos tomado desde fuera, y al hacerlo nos ha atraído algo que resultaba útil a nuestros propósitos en algún sentido. Es decir, desde el principio en España, en los finales del siglo XIX y principios del XX, hubo un interés por aplicar la Psicología, sin que fucra acompañada al mismo tiempo por una investigación propia y original.

Hay en esto una cierta continuidad con nuestra más antigua tradición. Nuestros primeros psicólogos renacentistas, como Luis Vives, y Juan Huarte de San Juan, son precisamente, como muchas veces se ha notado, dos hombres preocupados por la Psicología aplicada.

En efecto, Vives dijo en su "Tratado de Psicología" que no le preocupaba qué cra el alma, sino cómo funcionaba: "No es cosa que nos importe demasiado saber qué es el ama, aunque sí y en gran manera, saber cómo es y cuáles son sus operaciones" (Lib. I, c. 12). Por eso el libro de llamaba "Del alma y de la Vida", porque lo que importaba era justamente esa vida cuando el alma funciona. Luis Vives estaba preocupado por los problemas psicológicos porque, como buen humanista, estaba extremadamente interesado e implicado en la vida concreta del hombre en este mundo, en cómo formarle para la vida. Por eso le preocuaba la pedagogía, la educación, y estaba convencido de que la Psicología podía ayudar precisamente con un conocimiento del comportamiento de los hombres que posibilitara una aplicación más efectiva de los métodos de formación.

Esa aplicación de la Psicología a la educación la recoge tambićn como idea clave Juan Huarte de San Juan. También él piensa que para formar correctamente a los hombres hay que ver cómo son sus ingenios. Unos valen para unas cosas, otros valen para otras. Huarte de San Juan cuenta cierta estupenda historia, de cómo entraron tres jóvenes a los estudios, y comenzaron a mostrar grandes diferencias en sus habilidades: "Porque entamos tres compañeros a estudiar juntos latín, y el uno lo aprendió con gran facilidad, y los demás jamás pudicron componer una oración elegante. Pero pasados todos tres a dialéctica, el uno de los que no pudieron aprender gramática salió en las artes un águila caudal y los otros dos no hablaron palabra en todo el curso. $Y$ venidos todos tres a oir astrología, fue cosa digna de considerar que el que no pudo aprender latín ni dialéctica, en pocos días supo más que el propio maestro que nos enseñaba, y a los demás jamás nos pudo entrar" (cap. 1). Desde entonces, dice Huarte que empezó a pensar que habría que examinar a las personas y prepararlas para aquello para lo que estén capacitadas, lo que sería la única manera de reformar verdaderamente la sociedad.

Huarte tenía una concepción estrictamente aplicada a la teoría constitucionalista de la personalidad. El hombre tiene una estructura psicológica en función de su constitución psicofísica; si descubrimos cual es su verdadera capacidad entonces podremos dedicarlo a aquello para lo que resultará más útil y creativo; podremos transformar la sociedad. Huarte estaba convencido de que, para que una sociedad prosperara, hacían falta muchos hombres inteligentes; además, estaba convencido de que, como las mujeres son muy húmedas y los hombres muy secos, aquellas tendrán memoria pero no tienen inteligencia, estando ésta reservada para los hombres. Por eso, creía que era bueno enseñar a todos a fabricar varones y no mujeres y formuló ciertas reglas bastante convenientes a su juicio para lograr tan importante fin, formulando una incipiente 
eugenesia en su libro. ¡Hasta tal punto cra un hombre preocuado por la Psicología y por las técnicas aplicadas!

Estas ideas vienen a cuento aquí tan solo para que se vea que ha habido en los comienzos de la Psicología española una profunda, una muy fuerte implicación de las dimensiones prácticas de este conocimiento.

Sólo añadiré aquí cl recuerdo de Mariano Cubí, una figura que nunca se suele traer a colación, pero cuyo papel en la historia de nuestras ideas psicológicas no puede ser minusvalorado. Era un catalán que en el siglo XIX, después de haber ido a los Estados Unidos, volvió con la buena nueva del evangelio de la frenología, que fue propagando por todas partes. Su vida tiene un cierto sabor novelesco, topando en cierta ocasión con el Arzobispado de Santiago, manteniendo polémicas, y haciendo diagnósticos por las cárceles y las plazas. La frenología era un conocimiento aplicado al diagnóstico de las habilidades de la persona gracias al examen del cráneo de los individuos, y en ella nuevamente aparece la preocupación y el interés por una Psicología aplicada.

Cuando venimos al momento en que se inica un tímido desarrollo de la Psicología científica en nuestro país, a fines del siglo XIX; cuando se empieza a mencionar y a leer las obras de Wundt, de Maudsley o de Ribot, de todo lo que se llamaba en ese momento la Psicofísica, y se va a traer esa Psicología nueva como ciencia, se hace todo esto para apoyar otras cosas. ¿Cuáles?

Inicialmente, yo diría que la Psicología interesa para apoyar dos grandes cosas, la educación, y el ajuste social y la rehabilitación de deficientes y accidentados. Podriamos decir así que hay un primer periodo de introducción, de asentamiento de una inicial Psicología aplicada, donde habria habido una "Psicología sin Psicólogos", una psicología en manos de otras profesionales interesados en aplicar ciertas técnicas psicológicas a sus propios problemas.

El momento en que esa primera Psicología llega a nosotros, lo podríamos situar entre los años 1902 y 1920.

En efecto, en 1902 se creó una cátedra de Psicología en la Universidad de Madrid, que había de ocupar D. Luis Simarro, quien antes ya había disputado la cátedra de Histologia, de Madrid, a Ramón y Cajal. Simarro fue desde entonces catedrático de Psicología en la Facultad de Ciencias, y comenzó entonces un cierto esfuerzo por ir acercando la nueva Psicología a nuestro mundo intelectual, tratando de combinar dos grandes líneas, la Psicología fisiológica de los alemanes, y la Psicología asociacionista y descriptiva de los ingleses.

Ya desde ese primer momento se puede entrever lo que en nuestro país ha sido casi una constante: el no llegar a tener una escuela ortodoxa de Psicologia, porque siempre acabamos haciendo reajustes para que las cosas se arreglen de un modo un tanto ecléctico y el conflicto teórico se diluya al fin.

Tiene en esta época un valor casi simbólico el nombre de D. Luis Simarro, porque siendo el primer catedrático de Psicología, no debe haber dejado más de veinte artículos, de los cuales la mayoría son exposiciones de pequeños temas, uno es una conferencia de las teorías del sistema nervioso y otro una síntesis del proceso mediante el cual funciona la memoria, que él llamó "iteración", es decir, la asociación mediante la ley de ejercicio, algo bien concocido por todos en su tiempo. De modo que Simarro casi no escribió nada, y eso que escribió lo hizo de modo muy poco personal. Cierto 
que se interesó grandemente por los problemas sociales, y que sufrió mucho con el proceso a Ferrer, fundador en Barcelona de la escuela nueva; pero no acabó de hacer ciencia, aunque tuvo algunos discípulos, con los que había de echar a andar la psicología entre nosotros. Pero también aquí hay cierto extraño sino flotando sobre el grupo, como inmediatamente vamos a ver.

Uno de los grandes discípulos suyos fue Juan Vicente Viqueira, que a los veintitantos años se murió, tras haber estado en Alemania y en Francia, donde se había formado con Husserl, Bergson, y sobre todo, había estado con Gcorge Elias Müller, trabajando con él, y llegando a publicar un artículo sobre memoria y aprendizaje de sílabas en una revista especializada alemana. Posiblemente, él ha debido ser tal vez el único español que ha publicado un artículo en alemán sobre psicología antes de 1936. Pero con todo, este hombre muy pronto estuvo enfermo y no pudo hacer realmene Psicología, de modo creador.

Otro discípulo de Simaro ha sido Gonzalo Rodríguez Lafora, un médico psiquiatra, neuropatólogo, con una enorme atracción por la Psicología, pero que proyectó sobre ella una atención especialmente clínica y derivada, orientando su interés entonces hacia problemas clínicos en donde lo psicológico estaba implicado.

Lafora tuvo una extremada importancia en el desarrollo de esta Psicología española, pero, vuelvo a decirlo, con un sentido clínico. ¿Y qué preocupaba en la clínica en este primer momento? Por una parte, preocupaba en todo el mundo el diagnóstico de los niños anormales. Se había hecho un enorme descubrimiento, el de la eficacia de los tests, pues se había mostrado que el test de Binet y Simon permitía un conocimiento del individuo que lo situaba definiendo las condiciones de su posible capacidad educativa. El test se había convertido en el elemento simbólico de todo lo que un psicólogo podía ofrecer a la sociedad. Era pues preciso traer tests a España, y esto es lo que empezaron a hacer unos pocos hombres, Lafora entre ellos, para la reeducación de los niños "mentalmente anormales".

En estos primeros años que van desde principios de siglo al año 1920, en Madrid uno de los focos de interés, tal vez el dominante, lo podemos concentrar así en esa preocupación clínica por los niños mentalmente anormales y su psicopatologia.

En Barcelona las cosas han ido por un camino un poco distinto. En Barcelona hay un país mucho más industrializado, hay una problemática un poco diferente. Algunos grupos están interesados en promover una mejora social, y hacen el esfuerzo de promover un asesoramiento psicológico para la formación de aprendices que mejore la cualificación del personal laboral. Se funda en 1912 un Secretariado para la formación de aprendices que iba a hacer inmediatamente después orientación y diagnóstico profesional.

De este modo, pues, se va desarrollando en España un interés inicial hacia la psicología a lo largo de dos ejes: el de la aplicación al mundo escolar, (incluídos aquí los niños con problemas), y el de la utilización en el mundo industrial y laboral. Comenzamos a andar en Psicología trayendo ideas de fuera, y muy principalmente buscando tests y tratando de ajustarlos a las características de nuestra población.

Al mismo tiempo que se desarrolla esa preocupación por la Psicología comienza muy lentamente también a publicarse una revista, expresión de una cierta inquietud científica creativa en donde la Psicología tendrá un pequeño peso, los Archivos de 
Neurobiología que se publican desde 1920. Estamos en el fin de la primera ctapa de nuestra historia.

La preocupación que había surgido en estos años por aplicar una Psicología a ciertos problemas genera una segunda etapa que situariamos entre 1921 y 1936, a la que se podría caracterizar como la época de la formación de una psicotécnica española.

¿Por qué la llamo así? Porque la raíz y la preocupación aplicada de los años anteriores genera al fin unos primeros núcleos de institucionalización de la Psicología aplicada, y aquí ya empieza a haber personas que paulatinamente se van orientando hacia la Psicotecnia, entran en relación con el extranjero y son capaces en 1921 y luego en 1930 de organizar dos Congresos Internacionales de Psicotécnica, que tuvieron lugar en Barcelona.

La persona clave en este momento emerge en Barcelona y es Emilio Mira y López. Otra persona que quizá con menos brillo y un poco más a la sombra del Dr. Lafora trabaja en esta dirección es el Dr. José Germain. Emilio Mira y José Germain son dos médicos, pero son tal vez los primeros nombres en que uno podria poner al lado la calificación de "psicólogo", aunque quizá habría que ponerlo entre comillas, porque se ocuparon personalmente por hacer investigación para hacer una aplicación de métodos diagnósticos adecuados a la realidad de nuestro país en aquellos años.

Por fin el Dr. Germain, con Mercedes Rodrigo, pudieron publicar la primera adaptación del test de Terman en 1930, con el título de Pruebas de Inteligencia. Puso el prólogo al libro el Dr. Lafora, un prólogo donde dice unas cosas que deben ser tenidas aquí en cuenta. En efecto, el Dr. Lafora dice que el conocimiento psicológico ha llegado a una situación tal en los tests, que estos son utilizables ya con pequeños márgenes de error por personas no especializadas en los estudios psicológicos. La creencia que aquí late de que los elementos o instrumentos psicotécnicos eran tan objetivos, tan estruturados, tan perfectamente utilizables por cualquiera, desarrolla una convicción según la cual podríamos movernos cómodamente en una Psicología sin Psicólogos, un gravísimo malentendido que incluso todavía hoy tenemos que despejar. Ahí se plantea un problema muy grave: la indefinición o la mala definición del rol del psicólogo en nuestra sociedad, que aparecería como independiente de los instrumentos y las aplicaciones que crea, así como de la construcción teórica sobre la que se sustenta su práctica.

Hay que reconocer, sin embargo un creciente esfuerzo por ir dando cada vez mayor papel a la dimensión de la psicología como conocimiento, a su dimensión científica y a su dimensión institucional. Entre los años 1920 y 1930, en España se publican libros que permiten tener una información de primera mano del psicoanálisis, porque se tuvo aquí antes que en ningún otro sitio una colección de obras completas de Freud; también se traduce aquí en el mismo momento en que se publica en todo el mundo el libro sobre Los Reflejos Condicionados, de Pavlov, con un interesante prólogo del doctor Marañón. Al mismo tiempo se publican, a veces antes en francés, los primeros libros de Piaget, y se siguen publicando no sólo obras de psicoanálisis y de la Escuela de Ginebra, sino también la Psicología de la Gestalt, y precisamente Ortega desde la Revista de Occidente apoya la incorporación a España de las ideas de la fenomenología, de Brentano, Spranger o Dilthey. En suma, en esos años hay un admirable latido cultural en nuestro país, y vienen a dar conferencias figuras como Köhler, o Cannon, 
Michotte, o Piéron. Emilio Mira da muy pronto información sobre el conductismo americano en Iso "Archivos de Neurobiología", y va al Coingreso Internacional de 1929 en Yale con la descripción de un aparato que ha inventado para hacer diagnóstico de habilidades de conducción, el perceptotaquímetro. Esta es una primera versión de nuestra Psicología aplicada que ya va hacia afuera, es una primera psicotécnia que paulatinamente va encontrando cierto apoyo y cierto nivel institucional. De este modo se cmpieza a tejer entre nosotros la textura del conocimiento conceptual de lo que estaba haciendo la Psicología occidental de los años veinte, con unos desarrollos dentro del país originalmente centrados en la Psicología aplicada.

De todas las maneras, esa Psicología que empieza a institucionalizarse sufre una crisis total con la guerra civil. Tenemos que ser conscientes de que la guerra civil supuso un corte abrupto y gravísimo al desarrollo de una ciencia normal que paulatinamente iba incorporándose a nuestra cultura.

Como consecuencia de la tragedia, se produjo la emigración de muchos de los científicos que estaban en ese momento ocupados en investigaciones psicológicas. Emilio Mira, por ejemplo, acabó sus días en el Brasil, y toda la obra posterior a la guerra civil es una obra hecha en la emigración. No fue el único. Mercedes Rodrigo, el doctor Lafora, entre otros muchos, hubieron de partir.

Entre tanto, en España hubo un intento de sustituir la Psicología científica por una renovada Psicología escolástica, una Psicología filosófica montada fundamentalmente sobre las ideas medievales de Santo Tomas y tratando de hacer de esa Psicología un conocimiento filosófico, pero no una técnica de aplicación social. Frente a semejante intento, paulatinamente un pequeño grupo de personas que se reunieron en torno al Dr. Germain comenzaron un movimiento de recuperación de la Psicología científica, y, cosa curiosa, esa recuparación científica se hace, otra vez, al hilo de la Psicología aplicada.

En 1948, D. Jose Germain consigue que se cree un Departamento de psicología Experimental en el marco de un Instituto de Filosofía en lo que entonces era el Consejo Superior de Investigaciones Científicas, el máximo organismo de investigación. Allí se hace un pequeño espacio a la Psicología donde se sitúa el Dr. Germain con unos cuantos nombres más: José Luis Pinillos, Mariano Yela, Miguel Siguan, Francisco Secadas, el Padre Manuel Ubeda, Jesusa Pertejo, un grupo muy pequeño de personas pero muy decidido y entusiasta. Empiezan a recuperar los tests, de aptitudes mentales de Thurstone, adaptado por Secadas (AMPE), y se hace otro de personalidad (CEP) que incorpora dimensiones puestas de relieve por Eysenck, gracias al trabajo de Pinillos, que ha estado en contacto muy directo con el grupo de aquel, en Londres; y se desarrolla, en fín, un núcleo de investigación psicotécnica, en donde Yela trabaja y hace aplicaciones inmediatas de orientación y selección profesional, mientras Siguán se ocupa del TAT y Jesusa Pertejo estudia otras pruebas de orientación más dinámica. y hay un gran proyecto que tienen a todas estas personas en juego y es la selección de pilotos. Estados Unidos permiten estudiar y adaptar toda una serie de tests y se hace aqui una magnífica batería luego utilizada para la selección de esos profesionales. Esa tarea lleva un poco en vilo a la totalidad del Departamento, y asi nuevamente se recupera la Psicología científica precisamente desde esa dimensión aplicada. pero ya hacia 1950 , se han dado algunos pasos decisivos hacia la institucionalización de este 
conocimiento: se ha creado una revista, la Revista de Psicología General y Aplicada, se ha constituido ese Departamento, y se va a iniciar la formación en Psicología a nivel de la Universidad con una escuela de posgrado en Madrid, y luego otra en Barcelona.

Hubo además en los años cincuenta una incorporación de la psicología a la Universidad que permitió que hubiera catedráticos de Psicología en la Universidad formados ya dentro del grupo de Germain. Por eso pienso que desde 1950 empieza a incorporarse esa Psicología científica de manera más activa a las actividades culturales de nuestro país, en un proceso que cabría hacer llegar hasta 1968, con la aparición de una primera licenciatura universitaria de Psicólogo.

En este periodo hay la preocupación por ir logrando la institucionalización plena de la Psicología en España pero como ha dicho con mucha razón Yela aquí se empezó no desde abajo sino desde el tejado: empezamos teniendo unas escuelas de especialización y profesionalización en Psicología, pero no teníamos una formación universitaria de pregrado, es decir, no teníamos una licenciatura. De esta manera, seguimos teniendo una situación en donde profesionales de otras especialidades se convertían en Psicólogos, y no había una creación de la Psicología desde dentro y de una manera original y propia. Hay toda una serie de editoriales de la Revista de Psicología General y Aplicada diciendo que es necesario hacer una Psicología independiente, empezando por hacer la Psicología desde la Universidad. En todo caso, esa diplomatura en psicología sin embargo ya fue un paso porque, si hasta aqui habriamos tenido una Psicología sin Psicólogos, comenzábamos por lo menos ahora ya a tener un Psicología con Psicólogos, lo que no es poco.

Nuestro esfuerzo ha consistido, por tanto, en que la Psicología que empezó sin Psicólogos fuera crecientemente una Psicología con un Psicólogo cada vez mejor capacitado, cada vez mejor preparado desde la raíz, mejor dispuesto a enfrentarse con los problemas de la participación práctica y técnica.

Y en este sentido hemos vivido desde 1968 lo que yo llamaría un período de asentamiento profesional y científico creciente. Ha habido ciertos pasos que me parecen importantes y que han transformado la situación.

El primer factor que se ha producido ha sido la incorporación definitiva de la Psicología a la Universidad. Hay que tener presente que la Psicología surgió en Alemania y se desarrollo en Estados Unidos de una manera radicalmente original y creativa, muy posiblemente porque disputaron del mecanismo de investigación que la Universidad hace posible. Es decir, la Psicología comenzó en el mismo momento que tuvo una plataforma universitaria.

Aquí tuvimos esa inicial plataforma sólo desde 1968. Si se examina por ejemplo la evolución de las publicaciones en Psicología en las últimas décadas, aparece muy claramente que a partir de los años setenta se multiplica el número de revistas que se publican. La explicación más sencilla es que se ha multiplicado el número de grupos e investigadores que están trabajando. Por tanto, en este momento se produce un primer factor de transforamación, que es la incorporación de la psicología plenamente en la Universidad pero además con ello se lleva a cabo un desarrollo científico y conceptual. El hecho mismo de que la Universidad requiera de especializaciones, lo que ahora venimos llamando 'áreas de conocimiento', va produciendo de una manera casi mecánica la aparición de grupos que se concentran en áreas y problemas determinados. 
Así se va haciendo posible la división del trabajo, y por tanto el principio de especialización y de diversificación en la investigación.

Al incorporarse la Psicología a la Universidad no simplemente empieza a haber profesores de Psicología. Es algo mucho más importantc, es que se hacen posibles ciertas condiciones sociales objetivas que permiten la aparición creciente de la especialización, y la existencia social de la investigación en unas condiciones que antes no había; comienza aquí en cierto sentido un nuevo tipo de crecimiento.

Pero nuestro pais vive con una Universidad que fundamentalmente se piensa como un instrumento que da títulos profesionales. Por eso, inmediatamente se hacen patentes las consecuencias de la actual masificación universitaria, de tan graves y negativos efectos.

¿Qué ocurre? Simplemente que mientras las escuelas de posgrado, pudieron dar salida a dos o tres miles de Psicólogos en quince años, en el mismo momento en que las Universidades ponen la especialización en Psicología surgen muchos miles de Psicólogos en muy poco tiempo, con todos los problemas que eso pueda entrañar.

El desarrollo de los años setenta significa un crecimiento estructural de la Psicología como conocimiento, y también como profesión. Tendrá un valor simbólico, si se quiere, pero lo cierto es que 1980 es el año en que se establece la primera Facultad de Psicología como tal y además se constituye un Colgio Oficial de Psicólogos, de modo que la dimensión profesional y la dimensión científica adquieren un horizonte distinto, lleno a un tiempo de posibilidades y de problemas.

¿Cuáles son los problemas que a mi modo de ver podríamos perfilar en esta nueva situación?

Hay unos problemas que corresponden a la Psicología como conocimiento y otros que corresponden a la Psicología como profesión.

Un problema de la Psicología como profesión es que la Psicología ha comenzado por sufrir la influencia de la ley normal del mercado. Se ha producido más producto que el que la demanda requería: podemos estar al borde de tener más Psicólogos de lo que en un primer momento la sociedad pucde absorber; podemos tener muchos más profesionales que puestos claramente definidos en el marco social, lo que da origen a problemas de carácter laboral.

En segundo lugar en este crecimiento muy rápido en la formación de Psicólogos, no se ha clarificado suficientemente cuál es el rol que el psicólogo puede desempeñar en el marco social al cual está destinado. Como consecuencia de ello, aparecen situaciones más o menos conflictivas. El psicólogo que va a dedicarse a la aplicación de sus conocimientos en un marco clínico, o industrial, y desde luego en lo educativo, se encuentra con que en esas áreas hay otros profesionales mas o menos implicados dentro de ellas respecto de los cuales, como su rol del psicólogo no está perfectamente clarificado entra en conflicto con una cierta desventaja, porque llega como profesional más nuevo que los otros a un campo que ya está en buena medida ocupado.

Y la cosa se complica cuando resulta que la demanda de Psicólogos tiende a ser todavía muy indiferenciada en nuestra sociedad, y en buena medida es una demanda genérica que con mucha frecuencia no exige particular preparación a los psicólogos que se ofrencen a atenderla. Mientras en buena medida al psicólogo académico le preocupa la Psicología como ciencia, y busca elevar el nivel de su conocimiento, al 
profesional le importa la aplicabilidad de los conocimientos, y a veces surgen problemas en el sentido de si una real orientación hacia la Psicología aplicada debería de convertirse en una preparación o formación casi exclusivamente tecnológica, orientada hacia la formación de un psicotécnico, corriéndose el riesgo de perder de vista la formación básica, y el conocimiento de los procesos básicos a que un psicólogo tiene que hacer frente.

Estamos corriendo el riesgo, a veces, de perder de vista que toda aplicación tiene que ser la aplicación de unos conocimientos generales, y de unas leyes generales del comportamiento, junto a técnicas muy concretas y específicas. Así, la tensión entre una formación muy orientada a una práctica muy concreta y la formación hacia los puros y generales principios de la ciencia, crea una tensión general, en el mundo occidental, frente a la cual tenemos que colocarnos si queremos entender la situación presente. En estos momentos, yo diría que es absolutamente necesario formar psicólogos con todo rigor científico y prepararlos para la aplicación de intervenciones múltiples en el marco social.

Es absolutamente necesario formar Psicólogos con rigor porque con mucha frecuencia el Psicólogo oscila entre aplicar conocimientos fundados en leyes científicamente establecidas o convertirse en un apoyo espiritual del paciente que prescinde de los elementos técnicos que pueden hacer de su intervención una rigurosa aplicación de conocimientos sólidamente fundados. La preocupación por ayudar y modificar la situación del paciente o la persona que le consulta, utiizando en buena medida sólo el sentido común, puede hacer cada vez más problemático a los ojos de los demás el valor de la ciencia psicológica que se pretende aplicar, y eso es un riesgo gravísimo que el psicólogo tiene que evitar. La Psicología no es el puro sentido común, sino un conocimiento técnico de los comportamientos.

La formación de los psicólogos tiene que ser rigurosa, pero tiene que ser pensada de cara a una aplicación múltiple; es a mi modo de ver un peligro muy grave pensar que hay puras aplicaciones clínicas o educativas o industriales, como si el comportamiento estuviera en esos momentos definido por el contexto sin mas. En muchos casos, en muchísimos casos, el psicólogo tiene que operar con los procesos motivacionales, los procesos cognitivos, las dimensiones actitudinales sociales, o las dimensiones de personalidad, y son esos procesos los que en buena medida están condicionando el tipo de intervención que hace falta hacer. Esta, en muchos casos, es de carácter genérico, con una aplicabilidad casi idéntica en los marcos sociales de tipo educativo o en los de tipo industrial, como las técnicas de intervención de la mofificación de conducta han puesto de manifiesto. En suma, que es mucho mas importante el tipo de problemas a los cuales hay que hacer frente que la vieja concepción de área o de especialización contextual en donde el comportamiento tiene lugar.

Quiero decir con esto que en este sentido me parece absolutamente necesaria una auténtica compenetración entre las dimensiones científicas y académicas y las dimensiones profesionales de la Psicología en nuestro país.

Yo creo que el psicólogo y la Psicología en España hoy tienen un horizonte intelectual absolutamente comparable y homogéneo con el que tienen en otros países. Tenemos en estos momentos una literatura psicológica producida en España que da cuenta practicamente de los mismo problemas y el mismo horizonte de investigación 
que pueda tener la Psicología francesa, la Psicología inglesa, o la Psicología americana. No tendremos los mismo recursos ciertamente, pero lo que quiero decir es que estamos implantados ya en un nivel común intelectual y profesional.

Los psicólogos italianos, los psicólogos franceses, los psicólogos mejicanos evidentemente tienen problemas muy parecidos a los que estamos teniendo. No tenemos por qué plantearnos ningún tipo de pregunta especial, pero es importante que para plantear estos problemas pensemos con los datos reales de nuestra sociedad; quiero decir que no es posible que pensemos resolver la figura del psicólogo en nuestro país intentando copiar otros modelos externos, porque no es posible que se copie nada, sino que hay que buscar que la estructura que nosotros demos a nuestra profesión y a nuestra investigación esté adecuada a los datos de nuestra circunstancia.

En estos próximos años el psicólogo habrá de esforzarse por presentar un rol definido de tal manera que la sociedad inmediatamente lo reconozca y le apoye, lo busque y lo incorpore a su dinámica normal. Habrá de ser un rol en que, de modo esencial, estén ligadas las dos facetas esenciales que hemos visto en la Psicología, su dimensión como conocimiento y también como profesión. 